\title{
The role of SH3GL3 in myeloma cell migration/invasion, stemness and chemo-resistance
}

\author{
Ruoying Chen ${ }^{1}$, Hong Zhao ${ }^{2}$, Dan Wu ${ }^{1}$, Chen Zhao ${ }^{1}$, Weiling Zhao', Xiaobo Zhou ${ }^{1,3}$ \\ ${ }^{1}$ Department of Radiology, Wake Forest School of Medicine, Medical Center Boulevard, Winston-Salem, NC, USA \\ ${ }^{2}$ Department of Blood Transfusion, The Second Affiliated Hospital of Harbin Medical University, Harbin, Heilongjiang Province, \\ China \\ ${ }^{3}$ College of Computer Science and Software Engineering, Shenzhen University, Shenzhen, China \\ Correspondence to: Xiaobo Zhou, email: xizhou@wakehealth.edu \\ Keywords: CD138- cells, SH3GL3, migration/invasion, stemness, chemo-resistance \\ Received: January 31, $2016 \quad$ Accepted: September 12, $2016 \quad$ Published: September 24, 2016
}

ABSTRACT

Multiple myeloma (MM) is an incurable cancer characterized by clonal expansion of malignant plasma cells in the bone marrow and their egress into peripheral blood. The mechanisms of myeloma cells migration/invasion have remained unclear. Herein, we found SH3GL3 was highly expressed in the CD138-negative (CD138-) myeloma cells. The migration/invasion capability of CD138- cells was significantly higher than that in the CD138-positive (CD138+) cells. Silencing SH3GL3 using shRNA reduced myeloma cells migration/invasion. Conversely, overexpression of SH3GL3 increased myeloma cells migration/invasion. Moreover, SH3GL3 is also associated with the stemness and chemo-resistance of $\mathrm{CD}_{138^{-}}$myeloma cells. Elevated expression of stem cell and multi-drug resistant markers were seen in the myeloma cells with overexpressed SH3GL3; while knocking-down SH3GL3 reduced the expression of these markers. A marked increase in $p-P I 3 K$ and $p$-FAK was observed in the cells with overexpressed SH3GL3. To test if FAK/PI3K signaling pathway was involved in the SH3GL3-mediated myeloma cells migration, the cells transfected w/wo SH3GL3 cDNA were treated with FAK inhibitor 14 and PI3K inhibitor LY294002. Inhibition of FAK and PI3K attenuated SH3GL3-mediated migration /invasion. Our findings indicate that SH3GL3 plays an important role in myeloma cell migration/invasion, stemness and chemo-resistance. The SH3GL3-mediated myeloma cell migration/invasion is mediated by FAK/PI3K signaling pathway.

\section{INTRODUCTION}

Multiple myeloma (MM) is the second most prevalent hematologic malignancy. It is characterized by the clonal expansion of malignant plasma cells [1] and remains largely incurable in spite of the advent of several therapeutic strategies [2]. The clinical outcomes of MM patients are extremely heterogeneous, with survival ranging from several months to more than 15 years [3]. Even if MM patients may reach a complete remission initially with currently available chemo- and radio-therapy, most MM patients eventually developed relapsed disease after several years. A number of studies have suggested that a small fraction of cells responsible for disease relapse is capable of clonogenic growth and resistant to the therapeutic drugs $[4,5]$. This subpopulation is termed as multiple myeloma stem cells $[4,5]$. Recent studies indicated that clonogenic multiple myeloma cells lacked the expression of plasma cell marker CD138 [6] were enriched in CD138- CD19$\mathrm{CD}^{2} 8^{++}$plasma cells, while $\mathrm{CD} 19^{+} \mathrm{B}$ cells never formed MM colonies [7]. In contrast, Matsui et al. [4] found that CD $138^{-} \mathrm{CD}_{19}{ }^{+} \mathrm{CD} 27^{+}$cells isolated from the peripheral blood of MM patients were able to engraft in the NOD/ SCID mice and generate mature CD $138^{+}$myeloma cells.

Myeloma cell migration is very important for myeloma cell invasion and dissemination. However, the mechanism regulating cell migration remains unclear. Myeloma cells are thought to enter blood vessels in the periphery and then migrate to the bone marrow (BM) microenvironment by extravasting from the vascular endothelium. The interaction between myeloma cells and BM stromal cells promotes myeloma cell migration to secondary sites in the BM [8]. Studies have found that circulating malignant plasma cells were present in more than $70 \%$ of MM patients $[4,9]$. 
A number of studies have shown that several proteins participate in the migration of myeloma cells, such as insulinlike growth factor-1 (IGF-1) [10, 11], stromal cell-derived factor-1 $\alpha$ [12], wingless-ints (Wnt) [8], and integrin $\beta-7$ [13]. However, the complexity of migration process makes it likely that other molecules may also play critical roles.

In order to characterize the differences between CD138-positive and CD138-negtive cells, Yang et al. [14] performed gene expression profiling analysis and identified that a group of genes were upregulated in CD138- subgroup, including mammalian SH3-domain GRB2-like 3 (SH3GL3). The mammalian SH3GL3 is also known as endophilin A3 and extra eleven nineteen (EEN)-B2, and belongs to a small family of SH3 domain containing proteins [15]. Endophilins contain a Bin/ Amphiphysin/Rvs (BAR) domain, a variable region and an SH3 domain [15]. The BAR domain protein superfamily are important for cellular traffic. SH3 domains play critical roles in protein-protein interaction and are involved in signal transduction processes, such as the regulation of cancer cell migration/invasion and adhesion $[16,17]$. Endophilins also play important roles in receptor tyrosine kinase signaling, mitochondrial network dynamics, and synaptic vesicles retrieval [15].

Previous studies have shown that SH3GL3/ endophilin A3 is associated with the adaptor protein $\mathrm{Cb} 1-$ interacting protein of $85 \mathrm{~K}(\mathrm{CIN} 85)$. Ubiquitin ligase $\mathrm{Cb} 1$ recruits endophilin-CIN85 complex to mediate the internalization of receptor tyrosine kinases [18]. SH3GL3 is preferentially expressed in mammalian brain and testes, and can interact with Huntingtin exon 1 protein, dynamin, and synaptojanin [19]. Delic et al. [20] found that SH3GL3 was involved in glioma cell invasion and silence of SH3GL3 resulted in a reduction in the cumulative activity of matrix metalloproteins in these cells. To date, the specific function of SH3GL3 in cancer has yet to be addressed.

SH3GL3-associated signaling pathways are still poorly understood. A few of studies have focused on the role of endophilins in endocytosis. Aramaki et al. found that SH3GL3/endophilin A3 interacted directly with metastasis-associated protein 1 [21]. Endophilin3 -interacting protein 1 contains numerous SH3-domain binding sites, and it functions as an endocytic protein that affects energy homeostasis via its interaction with endophilins [22]. Endophilin A1 can be phosphorylated by Rho-associated protein kinase (ROCK) and leads to a reduction in EGFR endocytosis [23]. Phosphorylation of Endophilin A2 by a FAK/Src complex inhibited endophilin/dynamin interactions and abrogates endocytosis of MT1-MMP [17]. The interaction of Ataxin 2 with endophilin A1/A3, adaptor protein CIN85, and ubiquitin ligase c-CbI regulates endocytic receptor cycling [24]. The role of SH3GL3 in MM has not been investigated.

In this study, we found that CD138- myeloma stemlike cells had a higher migration and invasion capability. SH3GL3 was highly expressed in CD138 ${ }^{-}$myeloma cells.
Knocking-down SH3GL3 using shRNA reduced myeloma cell migration and invasion and decreased the stemness and chemo-resistance. In contrast, overexpression of SH3GL3 increased myeloma cell migration/invasion and enhanced the stemness and chemo-resistance of myeloma stem cells. Activation of FAK and PI3K were required for SH3GL3-increased myeloma cell migration/invasion. Inhibition of FAK or PI3K attenuated SH3GL3-enhanced cell migration. Taken together, these findings reveal, at the first time, that the SH3GL3-induced cell migration/ invasion is mediated by FAK/PI3K signaling pathway.

\section{RESULTS}

\section{Characterization of CD138- isolated from U266 myeloma cells}

$\mathrm{CD}_{138^{+}}$and $\mathrm{CD} 138^{-}$cells were isolated from U266 myeloma cells using autoMACS and CD138 microbeads. CD138- cells account about $3-4 \%$ of the total number of cells. Oct4, Lin28A, Nanog and Sox-2 are pluripotencyassociated transcription factors that maintain the selfrenewal and pluripotency of embryonic stem cells. We measured the expression of Lin28A, Nanog, OCT4, and Sox2 in the CD138 ${ }^{+}$and CD138 ${ }^{-}$cells using qRT-PCR. As shown in the Figure 1A, these genes were highly expressed in the CD138 ${ }^{-}$U266 myeloma cells, when compared with that in the $\mathrm{CD} 138^{+}$cells. The expressions of stem markers were also determined in other three myeloma cell lines (Supplementary Figure S2). It is known that clone formation ability reflects a self-renewal capacity, which is a characteristic of tumor stem cells. To examine the clonogenic capacity of both subpopulations, we carried out the soft agar clonogenic assay. As shown in the Figure 1B, CD138- cells had a higher clonogenic capacity than that in the $\mathrm{CD} 138^{+}$subpopulation. CD138 ${ }^{-}$cells from other three cell lines also displayed higher clonogenic capacity (Supplementary Figure S3). We also measured the sensitivity of both subpopulations to the therapeutic drug Bortezomib (BTZ). The CD138 ${ }^{+}$and CD138 ${ }^{-}$cells were treated with $0-10 \mu \mathrm{M}$ BTZ for 72 hours, and cell viability was determined using MTT assay. As shown in the Figure $1 \mathrm{C}$, the treatment CD138 ${ }^{+}$cells with 2, 5 and $10 \mu \mathrm{M}$ BTZ led to $16 \%, 51 \%$ and $54 \%$ reduction in cell viability, respectively, when compared to non-treated controls. In contrast, CD138 ${ }^{-}$cells were resistant to BTZ treatment. Incubating CD138- cells with $0-10 \mu \mathrm{M}$ BTZ for $72 \mathrm{~h}$ failed to affect cell viability (Figure 1D). Taken together, these results have demonstrated that $\mathrm{CD} 138^{-}$cells have the properties of stem cells and are resistant to BTZ treatment.

\section{CD138 $^{-}$cells exhibit a higher migration/invasion capability}

To evaluate the migration/invasion capacity of $\mathrm{CD}_{138^{-}}$and $\mathrm{CD} 138^{+}$cells, we measured cell migration 
and invasion using transwell assay. Comparing with the CD138 ${ }^{+}$cells, we observed a more than two-fold increase in the number of $\mathrm{CD} 138^{-}$cells migrated into the lower chamber (Figure 1E). Cell invasion was measured by assessing the migration of cells through matrigel-coated transwell filters overnight. Similarly, the number of CD138 cells invaded through matrigel was more than twice as much as the $\mathrm{CD} 138^{+}$cells (Figure 1F). Our data indicates that $\mathrm{CD} 138^{-}$cells have a higher migration and invasion capability.

\section{Overexpression of SH3GL3 enhances migration and invasion of myeloma cells}

The microarray analysis from Yang et al. [14] suggests that $\mathrm{CD} 138^{+}$and $\mathrm{CD} 138^{-}$cells have distinct gene expression profiles. We measured the mRNA levels of several genes in $\mathrm{CD} 138^{+}$and $\mathrm{CD} 138^{-} \mathrm{U} 266$ cells using qRT-PCR. We found that SH3GL3 was highly expressed in the $\mathrm{CD} 138^{-}$cells, and verified the protein level using western blotting in CD138+ and CD138-cells as shown in the Figure 2A. To test if SH3GL3 plays a role in myeloma cell migration and invasion, we first overexpressed SH3GL3 in a myeloma cell line. Human H929 myeloma cells expressed a relative low level of SH3GL3. We overexpressed SH3GL3 in this cell line. H929 cells were infected with lentiviral particles containing cDNA construct or empty vector. The mRNA and protein levels of SH3GL3 were determined using qRT-PCR and western blotting, respectively. A 3.8 fold increase of SH3GL3 mRNA level was seen in the SH3GL3 cDNA-infected cells, compared with the cells infected with empty vector (Figure 2B, bottom). The protein level of SH3GL3 was also increased in the SH3GL3 cDNA-infected cells (Figure 2B, top). H929 cell infected with SH3GL3 cDNA or empty vector were plated into the transwell inserts and the cells invaded into the bottom of the filter were counted. Overexpression of SH3GL3 resulted in a 3-fold increase in cell migration (Figure 2C). The number of cells invaded through the bottom of the matrigel membrane were also significantly elevated (Figure 2D). Our data indicated that overexpression of SH3GL3 enhanced migration and invasion of myeloma cells.

\section{Knocking-down SH3GL3 leads to a significant reduction in cell migration and invasion}

To further confirm the role of SH3GL3 in regulating myeloma cell migration and invasion, we knocked down the expression of SH3GL3 using SH3GL3 shRNA in U266 cells. U266 myeloma cells were infected with SH3GL3 shRNA lentiviral particles. As shown in the Figure 2E, infection of cells with SH3GL3 shRNA resulted in a $\sim 80 \%$ reduction in SH3GL3 expression, determined using qRTPCR (Figure 2E, bottom). The protein level of SH3GL3 was also markedly reduced (Figure 2E, top). As shown in the Figure 2F, knocking-down SH3GL3 led to a significant reduction in the number of migrated cells, when compared with the cells infected with scrambled shRNA lentiviral particles. The number of invading cells was also reduced significantly (Figure 2G). Thus, knocking-down SH3GL3 appears to play a critcal role in decreasing myeloma cell migration and invasion.

\section{Molecular mechanisms regulating MM cells migration induced by SH3GL3}

We then sought to determine the signaling pathways involving in the SH3GL3-activated migration of myeloma cells. Previous studies have reported the signaling pathways involved in the regulation of myeloma cell migration $[8,10-13]$. The focal adhesion kinase p125 (FAK) is a cytoplasmic tyrosine kinase and plays an important role in cell migration [25]. Phosphatidylinositol-3 kinases (PI3Ks) are a large family of lipid enzymes that can phosphorylate the $3^{\prime}-\mathrm{OH}$ group of phosphatidylinositol on the plasma membrane [26]. A study has demonstrated that the PI3K signaling pathway is involved in many different cellular processes such as motility, metabolism, cell survival and cell progression [27]. Recent results have indicated that the FAK and PI3K were involved in the migration of myeloma cells [10]. We, therefore, assessed the status of FAK activation in myeloma cells with overexpressed or silenced SH3GL3. As shown in Figure 3A, knocking-down SH3GL3 led to a significant reduction in the phosphorylated FAK protein level. In contrast, overexpression of SH3GL3 remarkably enhanced the phosphorylation of FAK (Figure 3B). We also analyzed the phosphorylated protein level of p85-PI3K. A similar pattern of changes in the phosphorylated protein levels of p85-PI3K was observed in the myeloma cells with reduced- or over- expression of SH3GL3 (Figure 3C and 3D).

Our data has shown that SH3GL3 regulated the activation status of FAK and p85-PI3K. Here, we investigated if the SH3GL3-activated cell migration and invasion were regulated through FAK/PI3K signaling. The cells infected with SH3GL3 shRNA or cDNA lentiviral particles were treated with either $10 \mu \mathrm{M}$ FAK inhibitor (FAK inhibitor 14) or $50 \mu \mathrm{M}$ PI3K inhibitor LY294002 for $1 \mathrm{~h}$ prior to seeding onto the transwell inserts. Figure 3E and $3 \mathrm{~F}$ show that preincubation of cells with FAK inhibitor 14 abolished the SH3GL3-promoted cell migration and invasion. Inhibition of PI3K using LY294002 also abrogated SH3GL3-induced migration and invasion in myeloma cells (Figure $3 \mathrm{G}$ and $3 \mathrm{H}$ ). These results indicated that FAK and PI3K were key mediators in the SH3GL3activated myeloma cell migration and invasion.

To determine the effect of FAK on the downstream signaling proteins, U266 cells were treated with FAK inhibitor and the related proteins were analyzed using western blotting. As shown in the Figure 4A, inhibition of FAK resulted reduction in the protein levels of $p$-PI3K, 
$p$-Src, $p$-RhoA, $p$-ROCK1 and $p$-P38. We didn't see any changes on other signaling molecules, such as ERK and AKT (data not shown). Our data indicated that PI3K, Src, RhoA, ROCK1 and P38 are involved in the FAK signaling. To determine the effect of SH3GL3 on FAK signaling pathway, the total and phosphorylated protein levels in the cells with infected shRNA or cDNA of SH3GL3 were compared with the non-infected counterparts. As shown in the Figure 4B, knocking-down SH3GL3 led to a reduction in the protein levels of $p$-Src, $p$ - RhoA, $p$-P38 and $p$-ROCK1. Conversely, overexpression of SH3GL3 enhanced the activation of these proteins (Figure 4C).

Based on the results that we obtained above and the published signaling pathways for FAK, we proposed a potential mechanism associated with the SH3GL3activated migration and invasion in the myeloma cells as shown in the Figure 5.

\section{Overexpression of SH3GL3 promotes myeloma cell stemness and enhances the expression of drug-resistant proteins}

To test whether SH3GL3 was indeed the driver rather than an associated phenomenon of stem cell features and drug resistance, we measured the expression of stem cell markers (Nanog, OCT4, Sox2 and Lin28A) in the H929 cells infected with SH3GL3 cDNA and empty vector using qRT-PCR. Overexpression of SH3GL3 led to a marked increase in the expression of Nanog, OCT4 and Sox2, respectively (Figure 6A). In contrast, knockingdown SH3GL3 using shRNA suppressed the expression of these stem cell markers (Figure 6B). No changes were observed in the expression of Lin28A in the cells with either overexpressed or silenced SH3GL3, when compared with their corresponding controls (data not shown). We also investigated the involvement of SH3GL3 in drug resistance. We measured the expression of multi-drug resistant markers, including multi-drug resistant protein 3 (ABCC3) and ATP-Binding Cassette Sub-Family B Member 1 (ABCB1). As shown in the Figure 6C and 6D, the expression of $\mathrm{ABCC} 3$ and $\mathrm{ABCB} 1$ were remarkably increased in the cells with overexpressed SH3GL3 (Figure 6C), and significantly reduced in the SH3GL3silenced U266 cells (Figure 6D). We subsequently examined the response of cells to BTZ. Myeloma cells infected with lentiviral particles containing SH3GL3 cDNA, shRNA or vectors were incubated with $5 \mathrm{nM} \mathrm{BTZ}$ for $72 \mathrm{~h}$ and cell viability was determined using MTT assay. Interestingly, overexpression of SH3GL3 resulted in an increase in cell viability when compared with the cells infected with empty vectors (Figure 6E). In contrast, the cells infected with SH3GL3 shRNA showed a reduction in cell viability compared with the scrambled control (Figure 6F). We also determined the role of SH3GL3 in cell response to melphalan and lenalidomide. U266 cells infected with SH3GL3 or scrambled shRNA were treated with $5 \mu \mathrm{M}$ melphalan or $10 \mu \mathrm{M}$ lenalidomide for $72 \mathrm{~h}$, respectively. Knocking-down SH3GL3 sensitized the U266 cells to melphalan, but not lenalidomide (Supplementary Figure S4). Taken together, these results suggest that SH3GL3 plays a potential role in the stemness and drug resistance of myeloma cells to BTZ and melphalan.
A

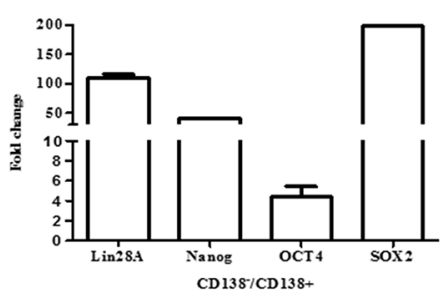

D

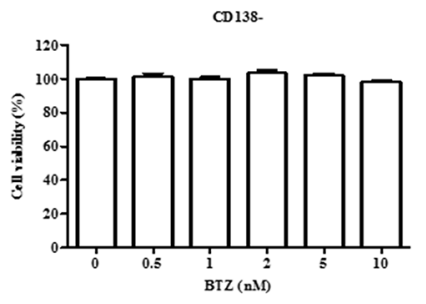

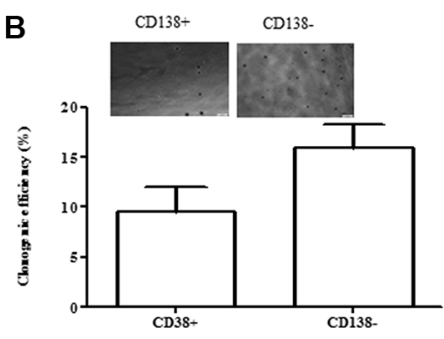

E

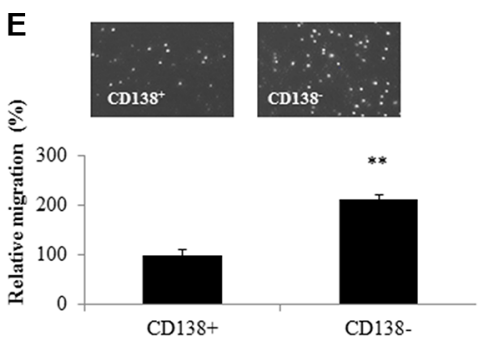

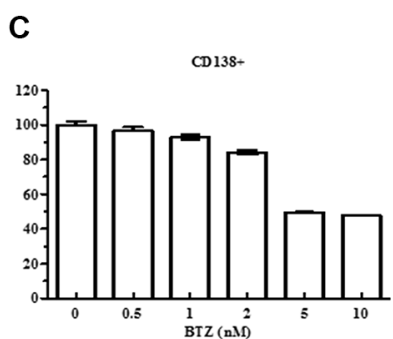

$\mathbf{F}$

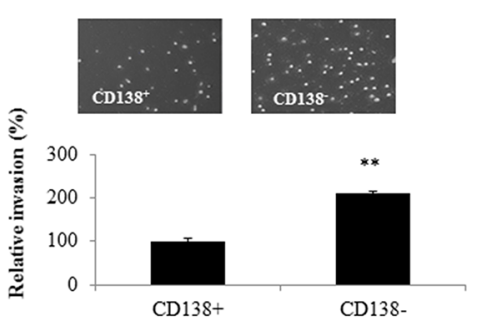

Figure 1: $\mathrm{CD}^{-138^{-}}$cells display the characteristics of stem cells and have greater migration and invasion capability. (A) The expression of stem cell markers including Lin28A, Nanog, OCT4 and Sox 2 was examined in the CD138 and CD138 U266 cells using qRT-PCR. (B) CD138 ${ }^{+}$and CD138 ${ }^{-}$U266 cells display different clonogenic capability. (C-D) MTT assay showed the responses of CD138 ${ }^{+}$and $\mathrm{CD} 138^{-} \mathrm{U} 266$ cells to the treatment with various concentration of BTZ. The relative value (\%) was calculated as the ratio of the number of treated cells and the number of untreated cells. (E) Transwell migration assay demonstrated the migration of CD138 $8^{-}$and CD138 $8^{+}$cells. (F) Transwell matrigel-coated invasion assay showed the invasion of $\mathrm{CD}_{138^{-}}$and $\mathrm{CD} 138^{+}$cells. The relative value (\%) was calculated as the ratio of the number of $\mathrm{CD} 138^{-}$and the number of CD138 ${ }^{+}$. The results are representative of 3 independent experiments and shown as mean $\pm \mathrm{SE}$, ** $p<0.01$. 


\section{DISCUSSION}

Most tumors are hierarchically organized and sustained by a subset of clonogenic cells that have selfrenewal potentials. These clonogenic cells can grow clonally into tumors and have potential to metastasize to other sites. Disease relapse indicates that the cells responsible for tumor regrowth are relatively drug resistant. Recent studies indicate that multiple myeloma contains heterogeneous cell types with different clonogenic potential [6]. The clonogenic myeloma cells exhibit properties of stem cells and are resistant to drug treatment $[4,28]$. CD138 is expressed in malignant and normal plasma cells, but not on virgin/ naive B cells, memory B cells, T cells, or monocytes [29]. Previous studies have shown that human myeloma cell lines and clinical specimens consist of only a minority of cells that lacked CD138 expression [6]. These CD138 ${ }^{-}$cells display more clonogenic potential in vitro and in vivo than $\mathrm{CD} 138^{+}$plasma cells and exhibit stem cell properties $[4,30]$. In the past several years, many efforts have been focused on clonogenic myeloma cells and their roles in myeloma initiation and relapse [6, 14]. However, the exact mechanism and their functional roles in the disease process are yet to be elucidated.
Though much progress in investigating clonogenic myeloma progenitor cells has been made, it is still debated whether $\mathrm{CD} 138^{-}$cells are myeloma stem cells. In our study, we isolated CD138- cells from myeloma cell line U266, NCI H929, RPMI8226, and MM1.S using autoMACS separator and CD138 microbeads, and measured the expression of stem cell markers and their clonogenic ability. We found that the CD138 ${ }^{-}$cells isolated from U266 cell line expressed a high level of Lin28A, Nanog, OCT4, and Sox2, and had a higher clonogenic potential compared with the $\mathrm{CD} 138^{+}$cells. The CD138 ${ }^{-}$cells isolated from other myeloma cell lines had also higher clonogenic potential. CD138- U266 myeloma cells were resistant to the BTZ. Furthermore, it has been reported that $\mathrm{CD} 138^{-}$cells could initiate tumor development and differentiate into $\mathrm{CD} 138^{+}$cells in vivo [31]. Moreover, CD138 ${ }^{-}$cells from a murine model of $\mathrm{MM}$ are quiescent and most invasive [32]. In our study, we also found that $\mathrm{CD} 138^{-}$cells exhibited a stronger invasion capability than $\mathrm{CD} 138^{+}$cells in vitro. Previous studies and our data suggest that $\mathrm{CD} 138^{-}$clonogenic myeloma cells are myeloma stem cells with high migration potential.

We have demonstrated the distinct expression of SH3GL3 in CD138 and CD138 ${ }^{+}$U266 myeloma cells
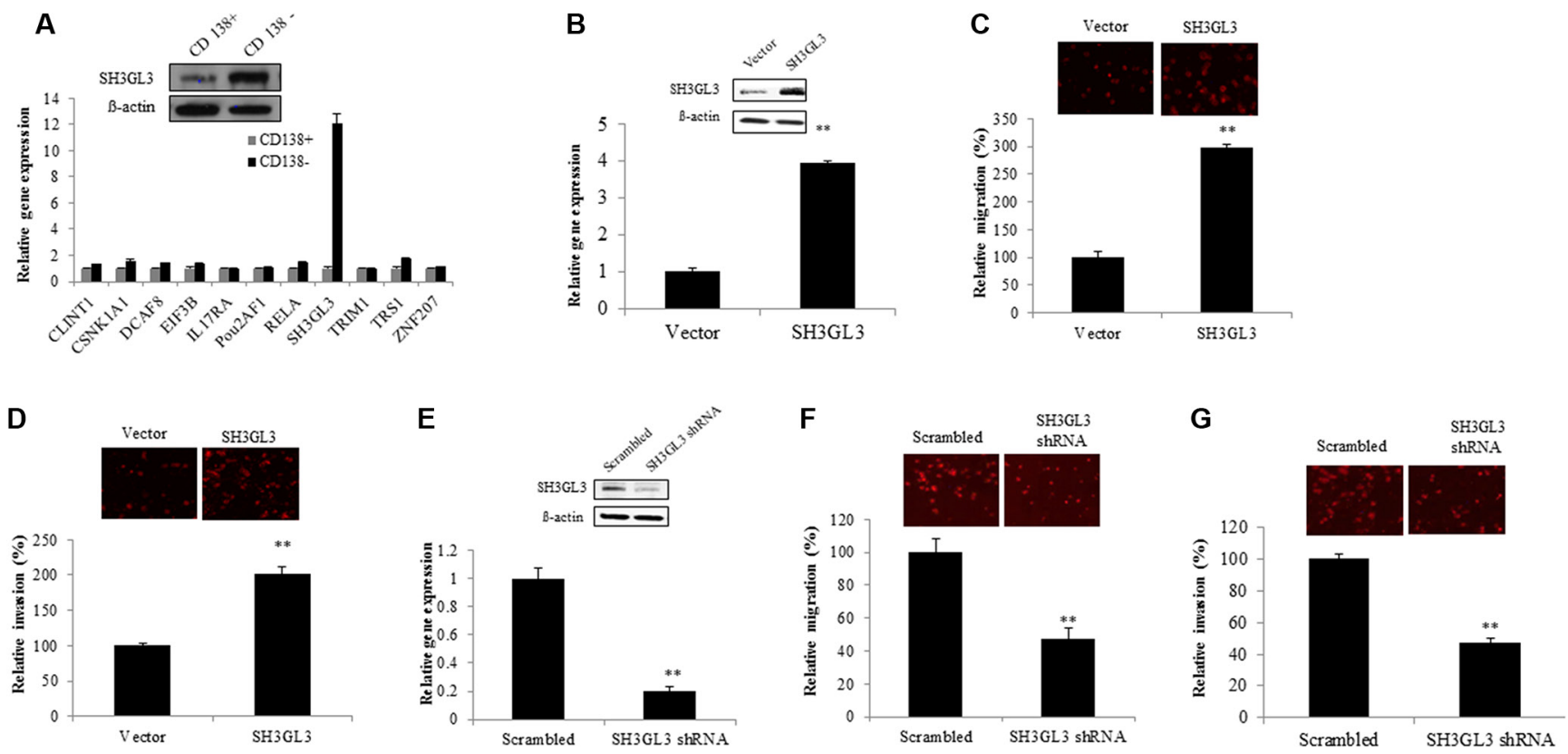

Figure 2: Overexpression of SH3GL3 enhances the migration and invasion of H929 myeloma cells; while knockingdown SH3GL3 leads to a significant reduction in U266 myeloma cells migration/invasion. (A) The expression of selected genes in the $\mathrm{CD}_{138^{+}}$and CD138- U266 cells was determined using qRT-RCR (lower panel). SH3GL3 was highly expressed in CD138 ${ }^{-}$ $\mathrm{U} 266$ cells and this gene was verified using western blotting at the protein level (upper panel). (B) The protein (upper panel) and mRNA (lower panel) levels of SH3GL3 in H929 cells infected with SH3GL3 cDNA lentiviral particles (expressed as SH3GL3) and empty vectors. (C) Transwell migration assay showed the number of migrated cells. (D) Transwell matrigel-coated invasion showed the number of invaded cells. (E) Western blotting and qRT-PCR analysis showed the protein (upper panel) and mRNA (lower panel) levels of SH3GL3 in the U266 cells infected with SH3GL3 shRNA lentiviral particles and scrambled vector. (F) Transwell migration assay showed the number of migrated cells. (G) Transwell matrigel-coated invasion showed the number of invaded cells. The relative value (\%) was calculated as the ratio of the number of treated cells and the number of vector control. Cells were labeled using Dil dye. The images were acquired with an Olympus microscope. The results are representative of 3 independent experiments and shown as mean $\pm \mathrm{SE}, * * p<0.01$. 
consistent with the data from microarray analysis [14]. Paino et al. [33] reported that $\mathrm{CD} 138^{++}$and $\mathrm{CD} 138^{\text {low }}$ have similar gene expression and genomic profiles. There are two differences between their study and ours. First, the isolation method they used was different from the one we used. Fluorescence-activated cell sorting was used by Paino et al., and autoMACS separator plus CD138 microbeads was used in our laboratory. Second, they analyzed CD138++ and CD138 ${ }^{\text {low }}$, but we analyzed CD $138^{+}$and $\mathrm{CD} 138^{-}$. Because CD138 ${ }^{-}$cells only account for a small subpopulation, it is more difficult to obtain enough CD138 ${ }^{-}$cells from MM patients. Therefore, gene profiling analysis using microarray has not shown a differential expression of SH3GL3 in the human MM specimen compared to the health control [34]. Delic et al. reported that SH3GL3 regulated glioma cell invasion, and knocking-down SH3GL3 reduced the activity of matrix metalloproteinase [20]. Iwakaki et al. [16] found that SH3 domains were critical to protein-protein interaction and regulated signal transduction process, such as cancer cell invasion. In our study, we found that increased numbers of cells migrated into the lower chamber when SH3GL3 was overexpressed, while few cells in the lower chamber when SH3GL3 was silenced. Our data suggest that SH3GL3 plays an important role in migration and invasion of myeloma cells.
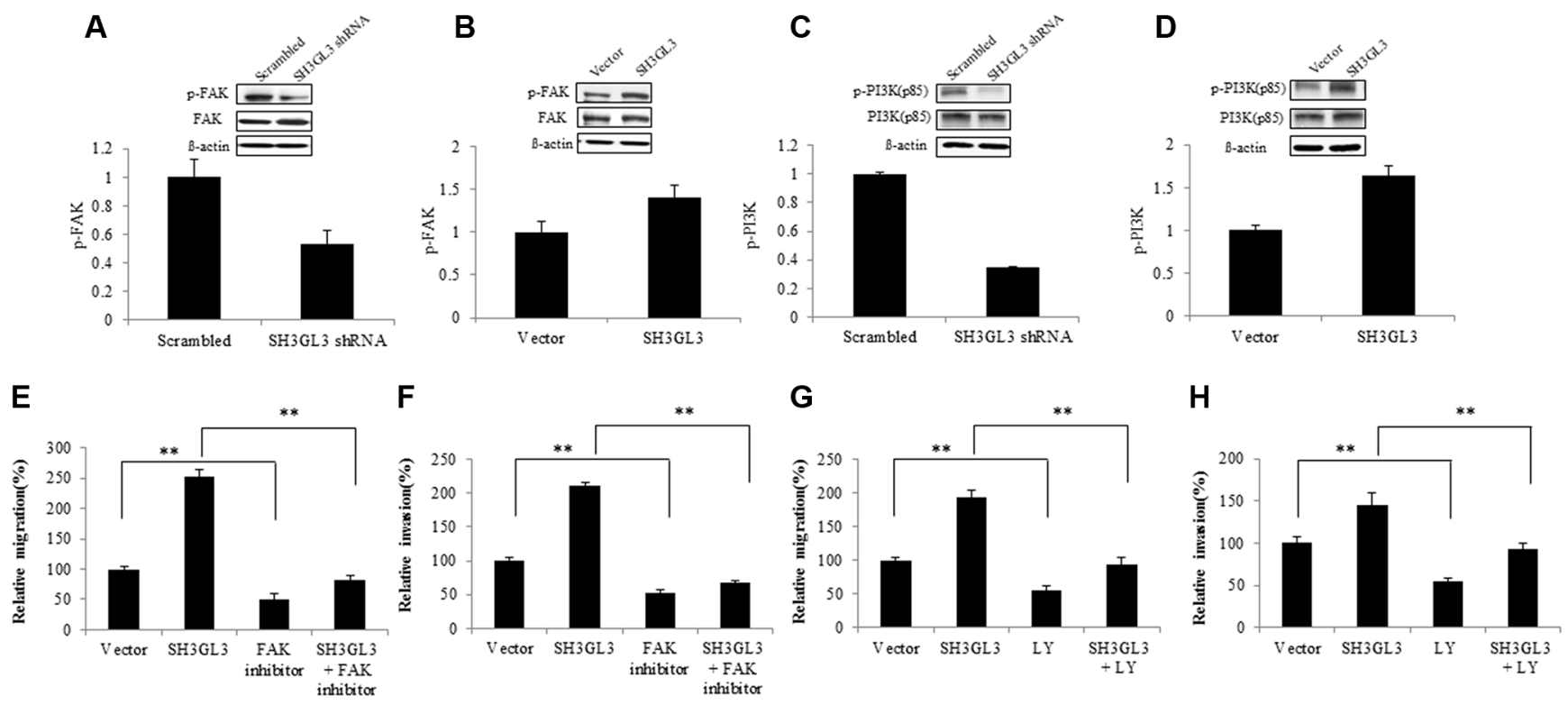

Figure 3: SH3GL3-induced myeloma cells migration/invasion is mediated through the activation of FAK/PI3K signaling pathway. (A-B) Western blotting analysis showed the protein level of FAK and $p$-FAk in the cells infected with SH3GL3 shRNA (A) or cDNA (B) lentiviral particles. (C-D) Wester blotting analysis showed the protein level of PI3K (85kd) and $p$-PI3K (85kd) in the cells infected with SH3GL3 shRNA (C) or cDNA (D) lentiviral particles. (E-F) H929 cells infected with SH3GL3 cDNA lentiviral particles were pretreated with FAK inhibitor $14(10 \mu \mathrm{M})$ for 1 hour and then cells were loaded into the insert in $300 \mu \mathrm{L}$ of serum-free RPMI 1640 medium. After 24 hours incubation at $37^{\circ} \mathrm{C}$, the cells in the lower chamber were counted. The relative migration was shown (E) and the relative invasion was shown (F). (G-H) H929 cells infected with SH3GL3 cDNA lentiviral particles were pretreated with PI3K inhibitor LY294002 $(50 \mu \mathrm{M})$ for 1 hour and then cells were loaded into the insert in $300 \mu \mathrm{L}$ of serum-free RPMI 1640 medium. After 24 hours incubation at $37^{\circ} \mathrm{C}$, the cells in the lower chamber were counted. The relative migration was shown $(\mathrm{G})$ and the relative invasion was shown $(\mathrm{H})$. The relative value was calculated as the ratio of the number of treated cells and the number of vector control. The results are representative of 3 independent experiments and shown as mean $\pm \mathrm{SE}, * * p<0.01$. 
defects in migration in vivo, suggesting the role of FAK in promoting cell migration [40]. In the present study, we found that the activation of FAK was stimulated by SH3GL3. Several FAK downstream signaling pathways have been known to involve in FAK-activated cell migration. Previous studies indicated that the FAK/ PI3K signaling pathway plays important roles in the regulation of cell migration [41, 42]. Reiske et al. found that PI3K inhibitor (LY294002) inhibited FAK-promoted migration in Chinese hamster ovary $(\mathrm{CHO})$ cells [41]. Our data showed that FAK inhibitor was able to suppress the activity of PI3K. Moreover, inhibition of FAK or PI3K reduced SH3GL3-activated cell migration and invasion. FAK can also increase cell migration through other pathways. Phosphorylation of FAK at Y397 creates a binding site for the $\mathrm{SH} 2$ domain of Src and triggers the complex formation of FAK-Src [43]. The FAK-Src complex can control cell shape, focal contact turnover and generation of traction forces events [44, 45]. Our results showed that FAK inhibition led to a significant inhibition of phosphorylated Src in the myeloma cells. It was reported that FAK overexpression could promote the p190RhoGEF (a ubiquitously expressed RhoA-specific GEF) tyrosine phosphorylation in Neuro-2a cells, which is associated with the enhanced activity of p21 RhoA. This event contributes to the regulation of cell migration $[46,47]$. ROCK, a downstream effector of RhoA, is also involved in cell migration [48]. RhoA/ROCK signaling pathway plays a crucial role in FAK activation. Therefore, we investigated the RhoA and ROCK as the downstream pathway of FAK. In our studies, the results showed that treating cells with FAK inhibitor reduced the protein levels of phosphorylated RhoA and ROCK, suggesting that they are the downstream signaling molecules of FAK. Additionally, we measured the activation of P38, Akt, and Erk. The activation of P38 was regulated by SH3GL3. Akt is the downstream signaling molecules of PI3K, so we also tested the status of Akt. No significant changes in the
A

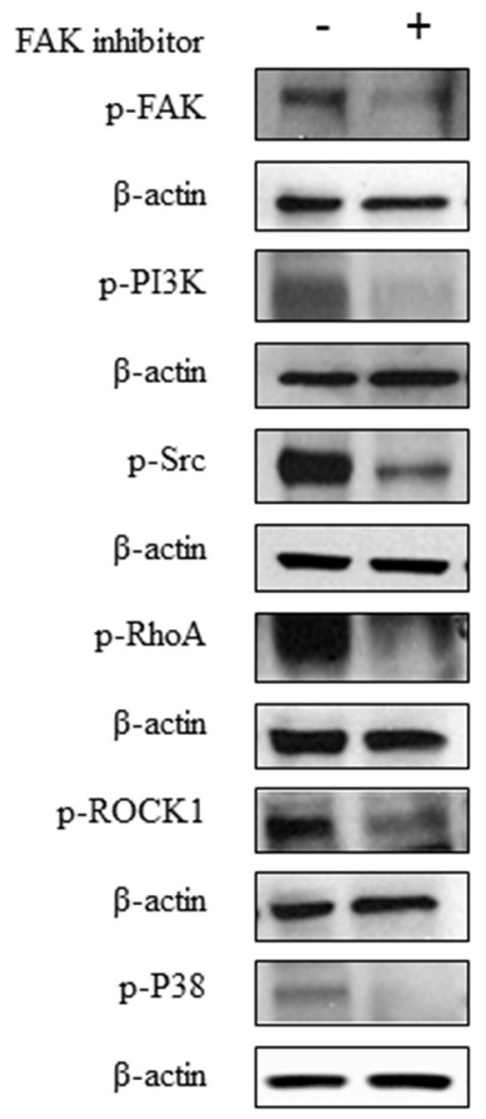

B

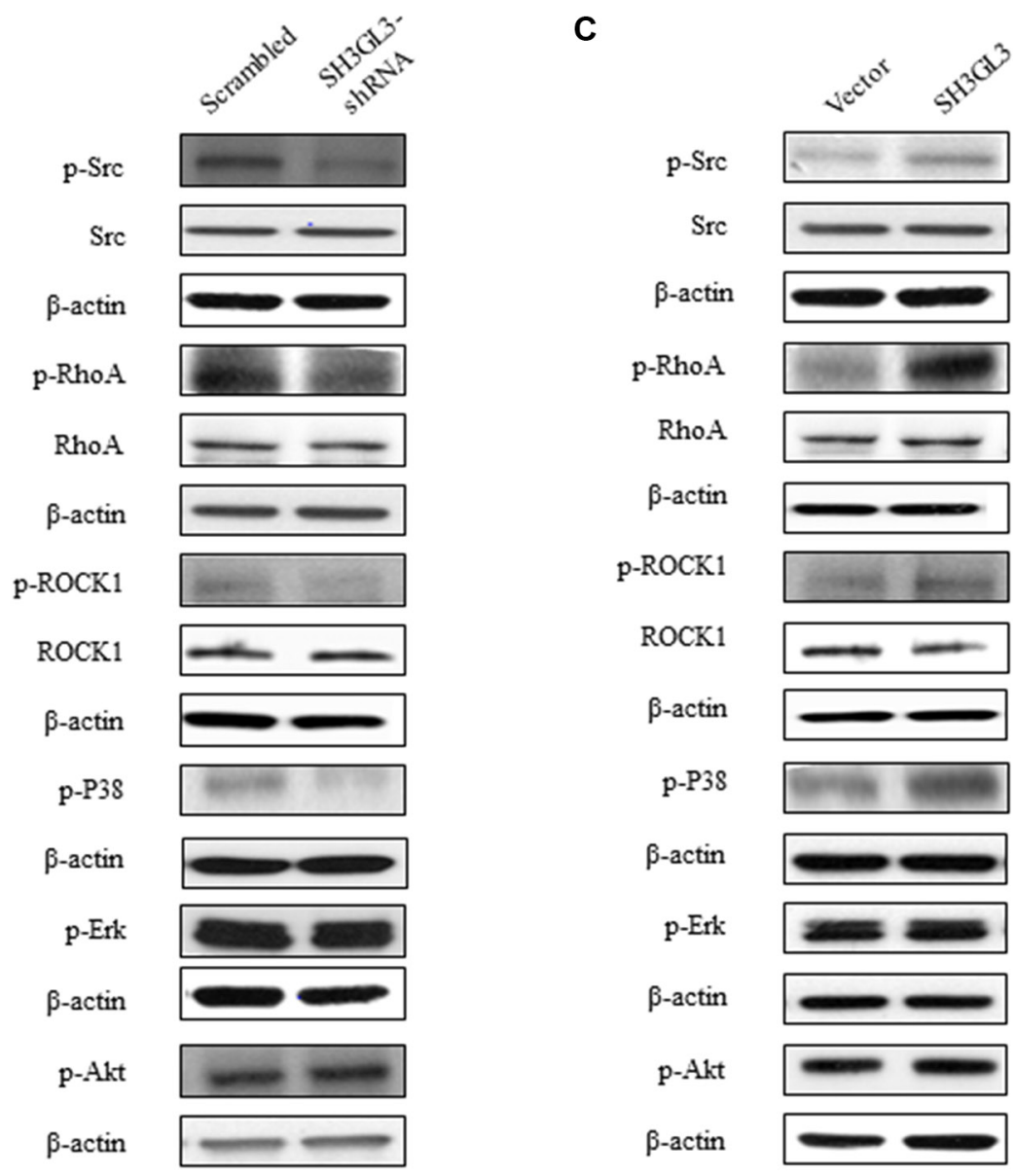

Figure 4: The activation status of Src, RhoA, ROCK1, P38, ERK, and AKT in the myeloma cells treated with FAK inhibitor or myeloma cells with suppressed and overexpressed SH3GL3. (A) The phosphorylated protein levels of FAK, PI3K, Src, RhoA, ROCK1, and P38 in the U266 cells treated with FAK inhibitor were decreased. (B) Representative blots showed the total and phosphorylated protein levels of Src, RhoA, ROCK1, P38, ERK and AKT in the U266 cells infected with SH3GL3 shRNA. (C) Representative blots showed the total and phosphorylated protein levels of Src, RhoA, ROCK1, P38, ERK and AKT in the U266 cells infected with SH3GL3 shRNA in the H929 cells infected with SH3GL3 cDNA. $\beta$-actin is loading control. 


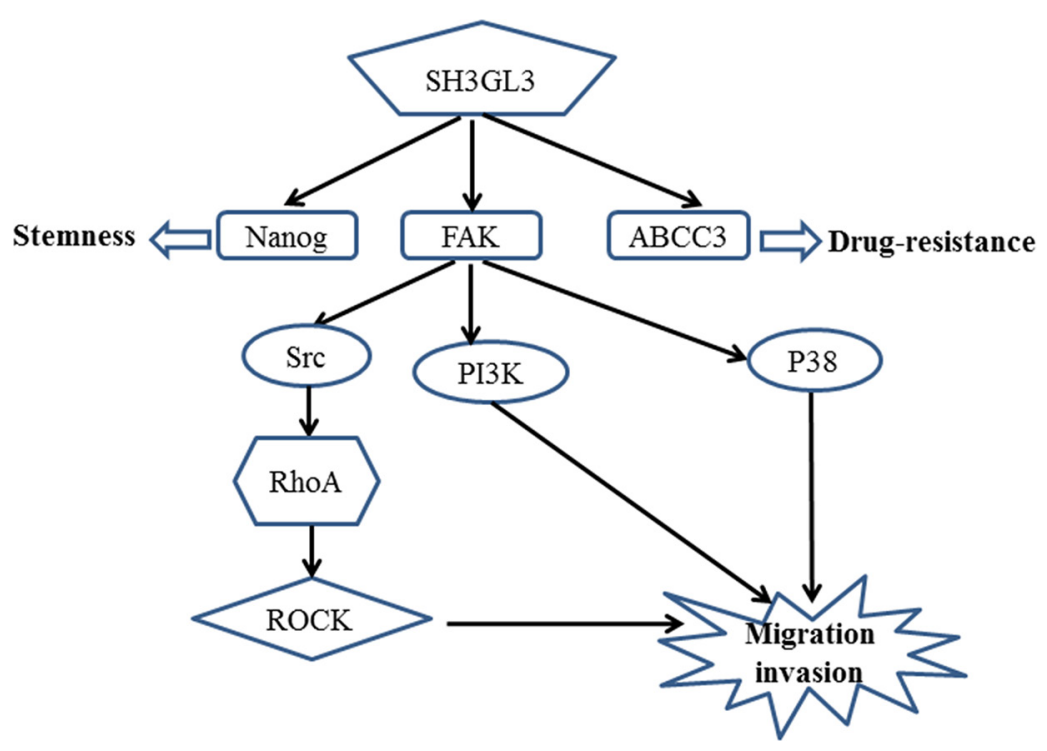

Figure 5: A schematic representation of the proposed mechanism for the SH3GL3-mediated migration/invasion, stemness and drug resistance of myeloma cells.
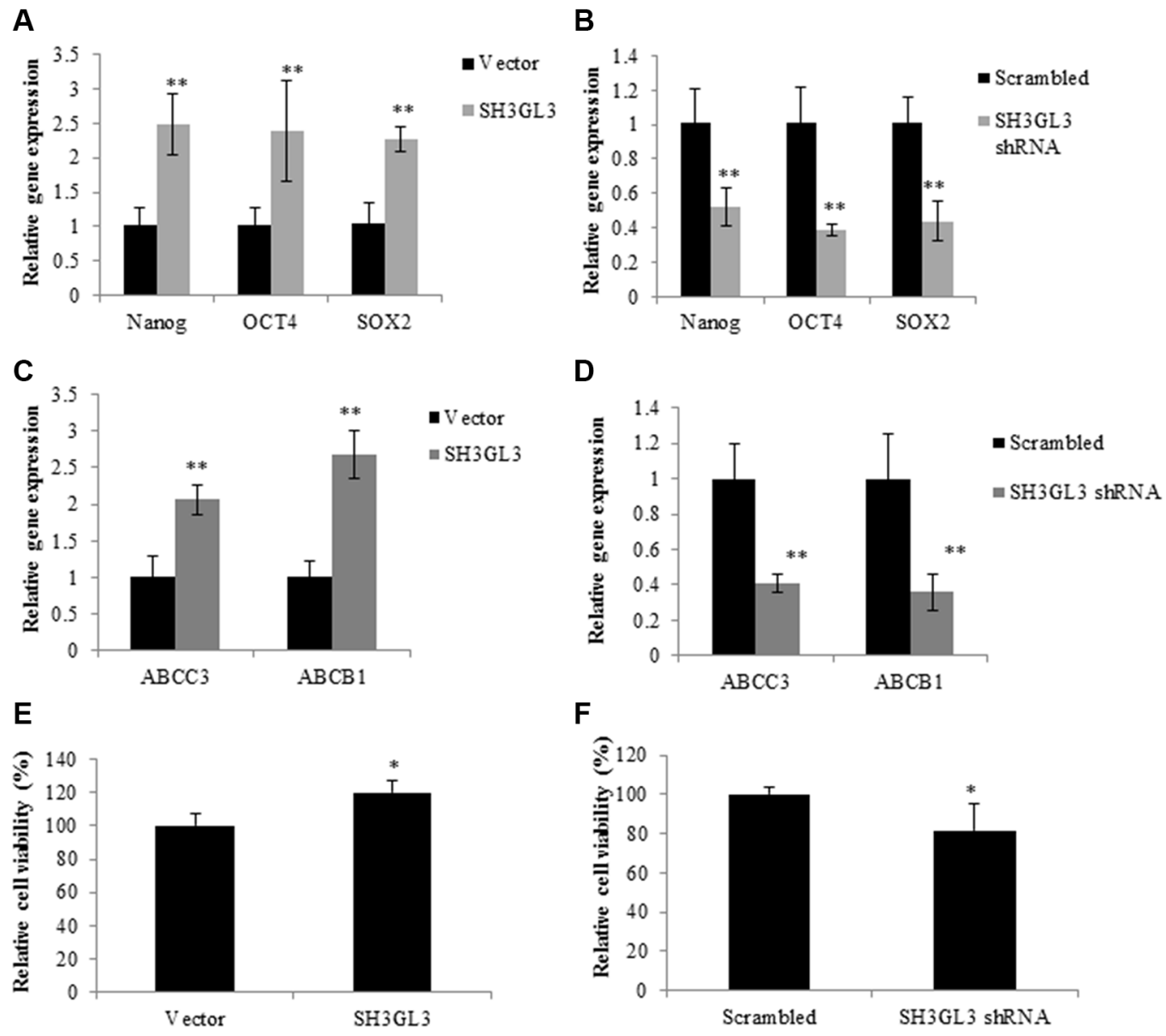

Figure 6: SH3GL3 regulates the expression of stem cell markers and drug resistance genes. Gene expression of Nanog, OCT4 and SOX2 was determined in the H929 cells infected with SH3GL3 cDNA (A) or U266 cells infected with SH3GL3 shRNA (B). qRT-PCR analysis showed the expression of the multi-drug resistance genes, ABCC3 and ABCB1, in the H929 cells infected with SH3GL3 cDNA $(\mathbf{C})$ or U266 with SH3GL3 shRNA (D). The cells infected with SH3GL3 cDNA (E) or shRNA (F) were treated with BTZ (10 nM) and cell viability was determined using MTT assay. The relative value was calculated as the ratio of treated cells and vector control. All experiments have been repeated 3 times, Mean $\pm \mathrm{SE},{ }^{*} p p<0.01,{ }^{*} p<0.05$. 
$p$-Akt was observed. Although Akt is viewed as a major downstream effector of PI3K, at least in physiological processes, several previous studies suggest that PI3K and Akt act independently of each other in cancers [49-52]. Recent studies have reported that Erk plays crucial roles in cell migration $[53,54]$. This protein was also detected in our study, and no significant changes in the $p$-Erk was observed. Our results showed that Akt and Erk were not involved in the SH3GL3-activiated cell migration. Based on our findings, we proposed a mechanism for the SH3GL3-promoted migration and invasion in myeloma cells as shown in the Figure 5. Our study indicates that SH3GL3-activated myeloma cell migration/invasion is regulated through the activation of FAK signaling pathways. Our current in vitro findings cannot be simply extrapolated to in vivo conditions. Therefore, in vitro observations need to be further confirmed using appropriate animal models to support the potential importance of SH3GL3 and related signaling molecules.

It is very important to target clonogenic myeloma cells, which are thought to be responsible for disease process and maintenance of MM [6]. Therapies against terminally differentiated plasma cells may lead to immediate clinical effects. The resistant stem cells may escape from the treatments. Therefore, selectively targeting myeloma stem cells could be prematurely abandoned. In summary, our findings provide the evidence of molecular mechanisms underlying the migration/ invasion of myeloma stem cells and offer potential insights into pathways that can be selectively targeted for the treatment of malignant MM disease. SH3GL3 may represent an attractive target for future research.

\section{MATERIALS AND METHODS}

\section{Cell culture}

The human myeloma cell lines U266 and H929 were purchased from the American Tissue Type Culture Collection (Manassas, VA), and cultured in RPMI 1640 medium (Thermo Scientific, Logan, UT) containing 10\% heat-inactivated fetal calf serum, $2 \mathrm{mM}$ L-glutamine, $100 \mathrm{U} / \mathrm{mL}$ penicillin, and $100 \mu \mathrm{g} / \mathrm{mL}$ streptomycin at $37^{\circ} \mathrm{C}$ in humidified $5 \% \mathrm{CO}_{2}$.

\section{$\mathrm{CD}^{138^{+}}$and $\mathrm{CD}^{-} \mathrm{8}^{-}$cell isolation}

$\mathrm{CD} 138^{+}$and $\mathrm{CD} 138^{-}$myeloma cells were isolated using autoMACS separator and CD138 microbeads (Miltenyi Biotec Inc., Auburn, CA) according to the manufacturer's instruction. Briefly, $2 \times 10^{7}$ cells were incubated with $40 \mu \mathrm{L}$ of CD138 microbeads for $15 \mathrm{~min}$ at $4^{\circ} \mathrm{C}$ and resuspended with $500 \mu \mathrm{L}$ incubation buffer. The cells were then transferred into a separation column, which was placed in a magnetic field in the autoMACS separator. The cells passing through the column were collected as
CD138 ${ }^{-}$portion. The magnetically labeled CD $138^{+}$cells were retained within the column and flushed out using $500 \mu \mathrm{L}$ incubation buffers. The purity of isolated CD138- cells was more than 94\% (Supplementary Figure S1).

\section{Clonogenic formation assay}

Colony formation was performed as described in [14]. Briefly, 5,000 myeloma cells were suspended in $0.35 \%$ agarose in $2 \times$ complete RPMI medium. The suspensions were placed onto a solidified base layer with $0.5 \%$ agarose in $2 \times$ complete medium in a 6 -well plate. The cells were cultured for three weeks and fresh medium was added to the culture plate every 3-4 days. All plates were imaged and colony numbers counted using Image J software. Only colonies containing more than 50 cells were scored.

\section{Lentiviral vector construction for SH3GL3 shRNA}

To determine the role of SH3GL3 in regulating myeloma cell migration and invasion, SH3GL3 shRNA and scramble sequences were designed using Invitrogen online shRNA software (http:/www.sirnawizard.com/ siRNA.php, Supplementary Table S1) and cloned into the pLVTHM vector. The constructs were then co-transfected with psPAX2 and $\mathrm{PMD}_{2} \mathrm{G}$ into $293 \mathrm{~T}$ packing cells using Lipofectamine 2000 (Invitrogen Life Technologies, Carlsbad, CA). After 48 hours, the packaged virus was collected and stored at $-80^{\circ} \mathrm{C}$ until use. The SH3GL3expressing lentiviral particles were obtained from GeneCopoeia (Rockville, MD). Myeloma cells were infected with SH3GL3 shRNA or SH3GL3-expressing particles in the presence of $8 \mu \mathrm{g} / \mathrm{mL}$ polybrene. The efficiency of SH3GL3 silence and overexpression in the myeloma cells were monitored by qRT-PCR and western blotting. The sequence 1 of shRNA got good results, and this shRNA was used in following assays.

\section{Real-time RT-PCR (qRT-PCR)}

Total RNA was extracted from myeloma cells using an RNeasy Mini kit (Qiagen, Valencia, CA) according to the manufacturer's instructions. First-strand cDNA was synthesized using the SuperScript III RT kit (Invitrogen, Carlsbad, CA). Quantitative real time PCR (qPCR) reactions were carried out on the 7500 Fast Real-time PCR system (Applied Biosystems, Foster City, CA). Briefly, qPCR amplification was performed with $100 \mathrm{ng}$ of cDNA in a $20 \mu \mathrm{L}$ reaction mixture containing $10 \mu \mathrm{L} 2 \times$ All-in-OneTM qPCR mix (GeneCopoeia, Rockville, MD), $300 \mathrm{nM}$ of upstream and downstream primers and nuclearfree water. PCR reaction was conducted with 1 cycle at $95^{\circ} \mathrm{C}$ for $10 \mathrm{~min}, 40$ cycles at $95^{\circ} \mathrm{C}$ for $15 \mathrm{~s}$ and $60^{\circ} \mathrm{C}$ for $1 \mathrm{~min}$, followed by dissociation curve analysis. The 
average $\Delta \Delta \mathrm{CT}$ and standard deviation were determined from three independent experiments. The expression of a gene was normalized with the expression of $\beta$-actin.

\section{Western blotting}

Myeloma cells were lysed with 1 X RIPA buffer supplemented with protease and phosphatase inhibitor cocktail (Roche Applied Science, Indianapolis, IN) and stored in aliquots at $-20^{\circ} \mathrm{C}$ until use. Twenty micrograms of cell lysates were mixed with an equal volume of Laemmli sample buffer, denatured by boiling, and separated by SDS-PAGE. The separated proteins were then transferred to a nitrocellulose membrane (BioRad, Hercules, CA). The membranes were blocked using 5\% non-fat dry milk for $1 \mathrm{~h}$ at room temperature and probed with antibodies overnight. After incubated with IgG horseradish peroxidase conjugated secondary antibodies (Cell signaling, Beverly, MA) for $2 \mathrm{~h}$ at room temperature, the immunoblots were developed using the enhanced chemiluminescence (ECL) reagent (Cell signaling, Beverly, MA) and visualized using a FluroChemQ processor (Proteinsimple, Santa Clara, CA).

\section{Cell migration and invasion assay using transwells}

For migration studies, the transwell inserts with $8 \mu \mathrm{m}$ pores in a 24-wells format (Coring Costar, Cambridge, MA) were used. One million cells were fluorescently labeled by incubation with $5 \mu \mathrm{L}$ of dialkyl carbocyanine membrane dye (Dil; Invitrogen) for $20 \mathrm{~min}$ at $37^{\circ} \mathrm{C}$. After removing the unbound dyes, $1 \times 10^{5}$ cells in $300 \mu \mathrm{L}$ of serum-free RPMI 1640 medium were seeded into the upper chamber and $500 \mu \mathrm{L}$ of RPMI 1640 medium with $100 \mathrm{ng} / \mathrm{mL} \mathrm{SDF}-1$ in the lower chamber. After $24 \mathrm{~h}$ incubation at $37^{\circ} \mathrm{C}$, the cells that migrated into the lower chamber were counted. Triplicates of each experiment were performed.

For the invasion assay, the transwell inserts were coated with $100 \mu \mathrm{L}$ Matrigel $(50 \mu \mathrm{g} / \mathrm{ml})$ for 4 hours. Dillabeled myeloma cells $\left(1 \times 10^{5}\right)$ in $300 \mu \mathrm{L}$ of serum-free medium were loaded into the upper chamber and $500 \mu \mathrm{L}$ of RPMI 1640 medium with $100 \mathrm{ng} / \mathrm{mL} \mathrm{SDF-1} \mathrm{in} \mathrm{the}$ lower chamber. After incubation for $24-48$ hours at $37^{\circ} \mathrm{C}$, the cells in the lower chamber was collected and counted.

For the experiments with specific inhibitors, myeloma cells were pretreated with the inhibitors for 1 hour and then loaded into the inserts. The cells in the control groups were treated with equal amount of vehicles. All images were acquired with an Olympus microscope.

\section{Cell viability assay}

Cell viability was determined using a modified thiazolyl blue tetrazolium bromide (MTT) (Acros
Organics, Thermo Fisher Scientific, New Jersey) method as described previously [55]. Briefly, 5000 cells/well were plated in 96-well plates and incubated overnight. The cells were treated w/wo bortezomib (various concentration $0.5 \sim 10 \mathrm{nM})$, melphalan $(5 \mu \mathrm{M})$ and lenalidomide $(10 \mu \mathrm{M})$ for 72 hours. Then $12.5 \mu \mathrm{L}$ of $5 \mathrm{mg} / \mathrm{mL}$ MTT reagent in PBS was added to each well and incubated for 4 hours in a $\mathrm{CO}_{2}$ incubator. Cells were then lysed by adding $50 \mu \mathrm{L}$ lysis buffer $(20 \% \mathrm{SDS}, 50 \% \mathrm{~N}, \mathrm{~N}, \mathrm{~N}$-dimethyl formamide (DMF), PH4.7) at $37^{\circ} \mathrm{C}$. The absorbance at $560 \mathrm{~nm}$ was measured using a spectrophotometer (Molecular Devices, Sunnyvale, CA).

\section{Statistical analysis}

Results are reported as the mean $\pm \mathrm{SD}$ for experimental groups performed in three replicate samples. Student $t$ test was applied to analyze the statistical significance of the differences between experimental groups. The $P$ values less than 0.05 were considered significant.

\section{ACKNOWLEDGMENTS}

The authors would like to thank Drs. Jing Su, Guangxu Jin, and Haiguo Sun for their comments and suggestions.

\section{CONFLICTS OF INTEREST}

Authors have no conflicts of interest to disclosure.

\section{GRANT SUPPORT}

This work was supported by a National Institute Health grant (NIH U01CA166886).

\section{REFERENCES}

1. Kyle RA, Rajkumar SV. Multiple myeloma. The New England journal of medicine. 2004; 351:1860-1873.

2. Dimopoulos M, Spencer A, Attal M, Prince HM, Harousseau JL, Dmoszynska A, San Miguel J, Hellmann A, Facon T, Foa R, Corso A, Masliak Z, Olesnyckyj M, et al. Lenalidomide plus dexamethasone for relapsed or refractory multiple myeloma. The New England journal of medicine. 2007; 357:2123-2132.

3. Barlogie B, Kyle RA, Anderson KC, Greipp PR, Lazarus HM, Hurd DD, McCoy J, Moore DF, Jr., Dakhil SR, Lanier KS, Chapman RA, Cromer JN, et al. Standard chemotherapy compared with high-dose chemoradiotherapy for multiple myeloma: final results of phase III US Intergroup Trial S9321. Journal of clinical oncology. 2006; 24:929-936.

4. Matsui W, Wang Q, Barber JP, Brennan S, Smith BD, Borrello I, McNiece I, Lin L, Ambinder RF, Peacock C, 
Watkins DN, Huff CA, Jones RJ. Clonogenic multiple myeloma progenitors, stem cell properties, and drug resistance. Cancer research. 2008; 68:190-197.

5. Ghosh N, Matsui W. Cancer stem cells in multiple myeloma. Cancer letters. 2009; 277:1-7.

6. Matsui W, Huff CA, Wang Q, Malehorn MT, Barber J, Tanhehco Y, Smith BD, Civin CI, Jones RJ. Characterization of clonogenic multiple myeloma cells. Blood. 2004; 103:2332-2336.

7. Hosen N, Matsuoka Y, Kishida S, Nakata J, Mizutani Y, Hasegawa K, Mugitani A, Ichihara H, Aoyama Y, Nishida S, Tsuboi A, Fujiki F, Tatsumi N, et al. CD138-negative clonogenic cells are plasma cells but not B cells in some multiple myeloma patients. Leukemia. 2012; 26:2135-2141.

8. Qiang YW, Walsh K, Yao L, Kedei N, Blumberg PM, Rubin JS, Shaughnessy J, Jr. Rudikoff S. Wnts induce migration and invasion of myeloma plasma cells. Blood. 2005; 106:1786-1793.

9. Bianchi G, Kyle RA, Larson DR, Witzig TE, Kumar S, Dispenzieri A, Morice WG, Rajkumar SV. High levels of peripheral blood circulating plasma cells as a specific risk factor for progression of smoldering multiple myeloma. Leukemia. 2013; 27:680-685.

10. Qiang YW, Yao L, Tosato G, Rudikoff S. Insulin-like growth factor I induces migration and invasion of human multiple myeloma cells. Blood. 2004; 103:301-308.

11. Tai YT, Podar K, Catley L, Tseng YH, Akiyama M, Shringarpure R, Burger R, Hideshima T, Chauhan D, Mitsiades N, Richardson P, Munshi NC, Kahn CR, et al. Insulin-like growth factor-1 induces adhesion and migration in human multiple myeloma cells via activation of beta1integrin and phosphatidylinositol 3'-kinase/AKT signaling. Cancer research. 2003; 63:5850-5858.

12. Alsayed Y, Ngo H, Runnels J, Leleu X, Singha UK, Pitsillides CM, Spencer JA, Kimlinger T, Ghobrial JM, Jia X, Lu G, Timm M, Kumar A, et al. Mechanisms of regulation of CXCR4/SDF-1 (CXCL12)-dependent migration and homing in multiple myeloma. Blood. 2007; 109:2708-2717.

13. Neri $\mathrm{P}$, Ren $\mathrm{L}$, Azab AK, Brentnall $\mathrm{M}$, Gratton $\mathrm{K}$, Klimowicz AC, Lin C, Duggan P, Tassone P, Mansoor A, Stewart DA, Boise LH, Ghobrial IM, et al. Integrin beta7mediated regulation of multiple myeloma cell adhesion, migration, and invasion. Blood. 2011; 117:6202-6213.

14. Yang Y, Shi J, Tolomelli G, Xu H, Xia J, Wang H, Zhou W, Zhou Y, Das S, Gu Z, Levasseur D, Zhan F, Tricot G. RARalpha2 expression confers myeloma stem cell features. Blood. 2013; 122:1437-1447.

15. Kjaerulff $\mathrm{O}$, Brodin L, Jung A. The structure and function of endophilin proteins. Cell Biochem Biophys. 2011; 60:137-154.

16. Iwasaki M, Homma S, Hishiya A, Dolezal SJ, Reed JC, Takayama S. BAG3 regulates motility and adhesion of epithelial cancer cells. Cancer research. 2007; 67:10252-10259.
17. Wu X, Gan B, Yoo Y, Guan JL. FAK-mediated src phosphorylation of endophilin A2 inhibits endocytosis of MT1-MMP and promotes ECM degradation. Dev Cell. 2005; 9:185-196.

18. Soubeyran P, Kowanetz K, Szymkiewicz I, Langdon WY, Dikic I. Cbl-CIN85-endophilin complex mediates ligandinduced downregulation of EGF receptors. Nature. 2002; 416:183-187.

19. Sittler A, Walter S, Wedemeyer N, Hasenbank R, Scherzinger E, Eickhoff H, Bates GP, Lehrach H, Wanker EE. SH3GL3 associates with the Huntingtin exon 1 protein and promotes the formation of polygln-containing protein aggregates. Molecular cell. 1998; 2:427-436.

20. Delic S, Lottmann N, Jetschke K, Reifenberger G, Riemenschneider MJ. Identification and functional validation of CDH11, PCSK6 and SH3GL3 as novel glioma invasion-associated candidate genes. Neuropathol Appl Neurobiol. 2012; 38:201-212.

21. Aramaki Y, Ogawa K, Toh Y, Ito T, Akimitsu N, Hamamoto H, Sekimizu K, Matsusue K, Kono A, Iguchi H, Takiguchi S. Direct interaction between metastasisassociated protein 1 and endophilin 3. FEBS letters. 2005; 579:3731-3736.

22. Trevaskis J, Walder K, Foletta V, Kerr-Bayles L, McMillan J, Cooper A, Lee S, Bolton K, Prior M, Fahey R, Whitecross K, Morton GJ, Schwartz MW, et al. Src homology 3-domain growth factor receptor-bound 2-like (endophilin) interacting protein 1, a novel neuronal protein that regulates energy balance. Endocrinology. 2005; 146:3757-3764.

23. Kaneko T, Maeda A, Takefuji M, Aoyama H, Nakayama M, Kawabata S, Kawano Y, Iwamatsu A, Amano M, Kaibuchi K. Rho mediates endocytosis of epidermal growth factor receptor through phosphorylation of endophilin A1 by Rho-kinase. Genes Cells. 2005; 10:973-987.

24. Nonis D, Schmidt MH, van de Loo S, Eich F, Dikic I, Nowock J, Auburger G. Ataxin-2 associates with the endocytosis complex and affects EGF receptor trafficking. Cellular signalling. 2008; 20:1725-1739.

25. Leventhal PS, Shelden EA, Kim B, Feldman EL. Tyrosine phosphorylation of paxillin and focal adhesion kinase during insulin-like growth factor-I-stimulated lamellipodial advance. J Biol Chem. 1997; 272:5214-5218.

26. Martini M, De Santis MC, Braccini L, Gulluni F, Hirsch E. PI3K/AKT signaling pathway and cancer: an updated review. Ann Med. 2014; 46:372-383.

27. Vanhaesebroeck B, Guillermet-Guibert J, Graupera M, Bilanges B. The emerging mechanisms of isoform-specific PI3K signalling. Nat Rev Mol Cell Biol. 2010; 11:329-341.

28. Brennan SK, Wang Q, Tressler R, Harley C, Go N, Bassett E, Huff CA, Jones RJ, Matsui W. Telomerase inhibition targets clonogenic multiple myeloma cells through telomere length-dependent and independent mechanisms. PloS one. 2010; 5. 
29. Chilosi M, Adami F, Lestani M, Montagna L, Cimarosto L, Semenzato G, Pizzolo G, Menestrina F. CD138/syndecan-1: a useful immunohistochemical marker of normal and neoplastic plasma cells on routine trephine bone marrow biopsies. Mod Pathol. 1999; 12:1101-1106.

30. Gottesman MM, Fojo T, Bates SE. Multidrug resistance in cancer: role of ATP-dependent transporters. Nat Rev Cancer. 2002; 2:48-58.

31. Reghunathan R, Bi C, Liu SC, Loong KT, Chung TH, Huang G, Chng WJ. Clonogenic multiple myeloma cells have shared stemness signature associated with patient survival. Oncotarget. 2013; 4:1230-1240. doi: 10.18632/ oncotarget.1145.

32. Asosingh K, De Raeve H, Van Riet I, Van Camp B, Vanderkerken K. Multiple myeloma tumor progression in the 5T2MM murine model is a multistage and dynamic process of differentiation, proliferation, invasion, and apoptosis. Blood. 2003; 101:3136-3141.

33. Paino T, Sarasquete ME, Paiva B, Krzeminski P, SanSegundo L, Corchete LA, Redondo A, Garayoa M, Garcia-Sanz R, Gutierrez NC, Ocio EM, San-Miguel JF. Phenotypic, genomic and functional characterization reveals no differences between CD138++ and CD138low subpopulations in multiple myeloma cell lines. PloS one. 2014; 9:e92378.

34. Gutierrez NC, Ocio EM, de Las Rivas J, Maiso P, Delgado M, Ferminan E, Arcos MJ, Sanchez ML, Hernandez JM, San Miguel JF. Gene expression profiling of B lymphocytes and plasma cells from Waldenstrom's macroglobulinemia: comparison with expression patterns of the same cell counterparts from chronic lymphocytic leukemia, multiple myeloma and normal individuals. Leukemia. 2007; 21:541-549.

35. Ridley AJ, Schwartz MA, Burridge K, Firtel RA, Ginsberg MH, Borisy G, Parsons JT, Horwitz AR. Cell migration: integrating signals from front to back. Science. 2003; 302:1704-1709.

36. Zhao X, Guan J-L. Focal adhesion kinase and its signaling pathways in cell migration and angiogenesis. Advanced drug delivery reviews. 2011; 63:610-615.

37. Golubovskaya VM, Cance WG. Focal adhesion kinase and p53 signaling in cancer cells. Int Rev Cytol. 2007; 263:103-153.

38. Owens LV, Xu L, Dent GA, Yang X, Sturge GC, Craven RJ, Cance WG. Focal adhesion kinase as a marker of invasive potential in differentiated human thyroid cancer. Annals of Surgical Oncology. 1996; 3:100-105.

39. Owens LV, Xu L, Craven RJ, Dent GA, Weiner TM, Kornberg L, Liu ET, Cance WG. Overexpression of the focal adhesion kinase (p125FAK) in invasive human tumors. Cancer research. 1995; 55:2752-2755.

40. Ilic D, Furuta Y, Kanazawa S, Takeda N, Sobue K, Nakatsuji N, Nomura S, Fujimoto J, Okada M, Yamamoto T. Reduced cell motility and enhanced focal adhesion contact formation in cells from FAK-deficient mice. Nature. 1995; 377:539-544.
41. Reiske HR, Kao SC, Cary LA, Guan JL, Lai JF, Chen HC. Requirement of phosphatidylinositol 3-kinase in focal adhesion kinase-promoted cell migration. J Biol Chem. 1999; 274:12361-12366.

42. Shen TL, Guan JL. Differential regulation of cell migration and cell cycle progression by FAK complexes with Src, PI3K, Grb7 and Grb2 in focal contacts. FEBS letters. 2001; 499:176-181.

43. Mitra SK, Schlaepfer DD. Integrin-regulated FAK-Src signaling in normal and cancer cells. Curr Opin Cell Biol. 2006; 18:516-523.

44. Mitra SK, Hanson DA, Schlaepfer DD. Focal adhesion kinase: in command and control of cell motility. Nat Rev Mol Cell Biol. 2005; 6:56-68.

45. Schlaepfer DD, Mitra SK, Ilic D. Control of motile and invasive cell phenotypes by focal adhesion kinase. Biochim Biophys Acta. 2004; 1692:77-102.

46. Zhai J, Lin H, Nie Z, Wu J, Canete-Soler R, Schlaepfer WW, Schlaepfer DD. Direct interaction of focal adhesion kinase with p190RhoGEF. J Biol Chem. 2003; 278:24865-24873.

47. Chikumi H, Fukuhara S, Gutkind JS. Regulation of G protein-linked guanine nucleotide exchange factors for Rho, PDZ-RhoGEF, and LARG by tyrosine phosphorylation: evidence of a role for focal adhesion kinase. J Biol Chem. 2002; 277:12463-12473.

48. Torsoni AS, Marin TM, Velloso LA, Franchini KG. RhoA/ ROCK signaling is critical to FAK activation by cyclic stretch in cardiac myocytes. Am J Physiol Heart Circ Physiol. 2005; 289:H1488-1496.

49. Stemke-Hale K, Gonzalez-Angulo AM, Lluch A, Neve RM, Kuo WL, Davies M, Carey M, Hu Z, Guan Y, Sahin A, Symmans WF, Pusztai L, Nolden LK, et al. An integrative genomic and proteomic analysis of PIK3CA, PTEN, and AKT mutations in breast cancer. Cancer research. 2008; 68:6084-6091.

50. Vasudevan KM, Barbie DA, Davies MA, Rabinovsky R, McNear CJ, Kim JJ, Hennessy BT, Tseng H, Pochanard P, Kim SY, Dunn IF, Schinzel AC, Sandy P, et al. AKTindependent signaling downstream of oncogenic PIK3CA mutations in human cancer. Cancer cell. 2009; 16:21-32.

51. Roper J, Richardson MP, Wang WV, Richard LG, Chen W, Coffee EM, Sinnamon MJ, Lee L, Chen PC, Bronson RT, Martin ES, Hung KE. The dual PI3K/mTOR inhibitor NVP-BEZ235 induces tumor regression in a genetically engineered mouse model of PIK3CA wild-type colorectal cancer. PloS one. 2011; 6:e25132.

52. Britschgi A, Andraos R, Brinkhaus H, Klebba I, Romanet V, Muller U, Murakami M, Radimerski T, Bentires-Alj M. JAK2/STAT5 inhibition circumvents resistance to $\mathrm{PI} 3 \mathrm{~K} / \mathrm{mTOR}$ blockade: a rationale for cotargeting these pathways in metastatic breast cancer. Cancer cell. 2012; 22:796-811.

53. Chen H, Zhu G, Li Y, Padia RN, Dong Z, Pan ZK, Liu K, Huang S. Extracellular signal-regulated kinase signaling pathway regulates breast cancer cell migration 
by maintaining slug expression. Cancer research. 2009; 69:9228-9235.

54. Huang C, Jacobson K, Schaller MD. MAP kinases and cell migration. Journal of cell science. 2004; 117:4619-4628.
55. Zhao W, Kridel S, Thorburn A, Kooshki M, Little J, Hebbar S, Robbins M. Fatty acid synthase: a novel target for antiglioma therapy. British journal of cancer. 2006; 95:869-878. 